\title{
Asymptotic direction for random walks in random environments
}

\author{
François Simenhaus ${ }^{1}$ \\ Université Paris 7, Mathématiques, case 7012, 2, place Jussieu, 75251 Paris, France \\ Received 16 December 2005; received in revised form 16 October 2006; accepted 31 October 2006 \\ Available online 12 January 2007
}

\begin{abstract}
In this paper we study the existence of an asymptotic direction for random walks in random i.i.d. environments (RWRE). We prove that if the set of directions where the walk is transient contains a non-empty open set, the walk admits an asymptotic direction. The main tool to obtain this result is the construction of a renewal structure with cones. We also prove that RWRE admits at most two opposite asymptotic directions.

(C) 2007 Elsevier Masson SAS. All rights reserved.

\section{Résumé}

Dans cet article, nous étudions l'existence d'une direction asymptotique pour les marches aléatoires en milieu aléatoire i.i.d. (RWRE). Nous prouvons que si l'ensemble des directions dans lesquelles la marche est transiente contient un ouvert non vide, la marche admet une direction asymptotique. La construction d'une structure de renouvellement avec cônes est le principal outil pour la preuve de ce résultat. Nous montrons aussi qu'une RWRE admet au plus 2 directions asymptotiques opposées.
\end{abstract}

(c) 2007 Elsevier Masson SAS. All rights reserved.

MSC: 60K37; 60F15

Keywords: Random walks in random environments; Asymptotic direction; Renewal structure

\section{Introduction and results}

In this paper, we give a characterization of random walks in random i.i.d. environments having an asymptotic direction. We first describe the model that we will use. Fix a dimension $d \geqslant 1$ (but think more particularly of the case where $d \geqslant 2$ because this work becomes obvious when $d=1)$. Let $\mathcal{P}_{+}$denote the $(2 d-1)$-dimensional simplex, $\mathcal{P}_{+}=\left\{x \in[0,1]^{2 d}, \sum_{i=1}^{2 d} x_{i}=1\right\}$. An environment $\omega$ in $\mathbb{Z}^{d}$ is an element of $\Omega:=\mathcal{P}_{+}^{\mathbb{Z}^{d}}$. For any environment $\omega, P_{x, \omega}$ denotes the Markov chain with state space $\mathbb{Z}^{d}$ and transition given by

$$
\begin{aligned}
& P_{x, \omega}\left(X_{0}=x\right)=1 \quad \text { and } \\
& P_{x, \omega}\left(X_{n+1}=z+e \mid X_{n}=z\right)=\omega_{z}(e) \quad\left(z \in \mathbb{Z}^{d}, e \in \mathbb{Z}^{d} \text { s.t. }|e|=1, n \geqslant 0\right),
\end{aligned}
$$

where $|\cdot|$ denotes the Euclidean norm in $\mathbb{Z}^{d}$.

E-mail address: simenhaus@math.jussieu.fr.

1 Partially supported by CNRS (UMR 7599 "Probabilités et Modèles Aléatoires"). 
For any law $\mu$ on $\mathcal{P}_{+}$, we define a random environment $\omega$ in $\mathbb{Z}^{d}$, random variable on $\Omega$ with law $\mathbb{P}:=\mu^{\otimes \mathbb{Z}^{d}}$. For any $x$ in $\mathbb{Z}^{d}$ and any fixed $\omega$, the law $P_{x, \omega}$ is called quenched law. The annealed law $P_{x}$ is defined on $\Omega \times\left(\mathbb{Z}^{d}\right)^{\mathbb{N}}$ by the semi-product $P_{x}:=\mathbb{P} \times P_{x, \omega}$. In this article, the law $\mu$ will verify the assumption of strict ellipticity

$$
\forall e \in \mathbb{Z}^{d} \text { s.t. }|e|=1, \mathbb{P} \text {-a.s. } \quad \mu\left(\omega_{0}(e)>0\right)=1,
$$

which is weaker than the usual uniform ellipticity (see Remark 1). $S^{d-1}$ denotes the unit circle for the Euclidean norm. For any $\ell$ in $\mathbb{R}^{d}$, we define the set $A_{\ell}$ of transient trajectories in direction $\ell$

$$
A_{\ell}=\left\{\lim _{n \rightarrow+\infty} X_{n} \cdot \ell=+\infty\right\},
$$

and for any $v$ in $S^{d-1}, B_{v}$ is defined as the set of trajectories having $v$ for asymptotic direction

$$
B_{v}=\left\{\lim _{n \rightarrow+\infty} \frac{X_{n}}{\left|X_{n}\right|}=v\right\} .
$$

This model is well studied in the one-dimensional case where many sharp properties of the walk are known. However in higher dimensions the behavior of the walk is much less well-understood. Particularly, the notion of asymptotic direction has been poorly studied. In this paper, we give a description of the class of walks having a unique asymptotic direction under the annealed measure (Theorem 1). It means that the walk is transient and escapes to infinity in a direction which has a deterministic almost surely limit. We also prove that under the annealed measure, a RWRE admits at most two opposite asymptotic directions (Proposition 1 and Corollary 1). The proofs are based on renewal structure as in [2] or [8].

The main difficulty to obtain an asymptotic direction for a transient walk is to control the fluctuations of the walk in the hyperplane transverse to transience direction. One way to control those fluctuations is to introduce the following assumption.

Assumption. $\ell$ in $\mathbb{R}_{*}^{d}$ verifies assumption $(\mathrm{H})$ if there exists a neighborhood $\mathcal{V}$ of $\ell$ such that

$$
\forall \ell^{\prime} \in \mathcal{V}, \quad P_{0}\left(A_{\ell^{\prime}}\right)=1 .
$$

When $(\mathrm{H})$ holds, we will note $\mathcal{V}$ the neighborhood given by the assumption.

The main purpose of this article is to prove the following theorem.

Theorem 1. The following three statements are equivalent

(i) There exists a non-empty open set $\mathcal{O}$ of $\mathbb{R}^{d}$ such that

$$
\forall \ell \in \mathcal{O}, \quad P_{0}\left(A_{\ell}\right)=1 .
$$

(ii) $\exists v \in S^{d-1}$ s.t.

$$
P_{0} \text {-a.s., } \quad \frac{X_{n}}{\left|X_{n}\right|} \underset{n \rightarrow \infty}{\longrightarrow} \text { v. }
$$

(iii) $\exists v \in \mathbb{R}_{*}^{d}$ s.t. $\forall \ell \in \mathbb{R}^{d}$

$$
\ell \cdot v>0 \Longrightarrow P_{0}\left(A_{\ell}\right)=1 \text {. }
$$

Using arguments similar to those applied in the proof of Theorem 1, we also show

Proposition 1. If $v$ and $v^{\prime}$ are two distinct vectors in $S^{d-1}$ such that $P_{0}\left(B_{v}\right) P_{0}\left(B_{v^{\prime}}\right)>0$ then $v^{\prime}=-v$.

An obvious consequence of this proposition is the following corollary.

Corollary 1. Under $P_{0}$, there are at most two asymptotic directions, in this case these two potential directions are opposite each other. 
The class of walks admitting an asymptotic direction has been poorly studied so far. Theorem 1 gives a characterization of this class but also leaves unsolved some important problems related to this notion. First, we would like to compare the ballistic class with the class of walks admitting an asymptotic direction. As shown in Remark 2, if a walk admits an asymptotic direction, it also satisfies a law of large numbers. However, the notion of asymptotic direction is of interest only in the non-ballistic case. Indeed, a non-degenerate velocity contains more information (direction and speed) than the asymptotic direction (direction only) whereas in the non-ballistic case the asymptotic direction gives an interesting information of the behavior which is not contained in the law of large numbers. It is known that in dimension 1, the class of walks admitting an asymptotic direction (here it simply means transient) but a degenerate velocity is non-empty. In higher dimension we have no example of such a walk and it might be possible that there is none.

The class of ballistic walks has been the subject of many recent articles. In the first one [8], the authors provide a strong sufficient drift condition to obtain a ballistic law of large numbers, Kalikow's condition. Later, Sznitman improves on sufficient conditions in different works, [6] is the more recent. He introduces in this paper the conditions $\left(T_{\gamma}\right)(\gamma \in(0,1])$ that we will not recall, and the condition $\left(T^{\prime}\right)$ defined as the realization of $\left(T_{\gamma}\right)$ for any $\gamma \in(0,1)$. According to Corollary 5.3 in [7], this condition is strictly weaker than Kalikow's criterion for $d \geqslant 3$ and Sznitman gives an effective criterion to check it, that is the weakest condition known to assure a ballistic behavior. It is also shown in (1.13) of [6] that $\left(T_{\gamma}\right)$ implies that the walk has an asymptotic direction (and so using Theorem 1 implies $(\mathrm{H}))$, it is then natural to ask if $(\mathrm{H})$ is strictly weaker than $\left(T_{\gamma}\right)$ or simply equivalent. This question is particularly interesting because $\left(T_{\gamma}\right)$ is equivalent to $\left(T^{\prime}\right)$ for $\gamma \in\left(\frac{1}{2}, 1\right)$ when $d \geqslant 2$, and it is conjectured that they are equivalent for any $\gamma \in(0,1)$ (see [6], in particular Theorem 2.4). An answer to this question could be a step toward comparing the ballistic class and the class of walks admitting an asymptotic direction and then would help us to solve the first problem above.

A third problem is that we could not find a criterion to check assumption $(\mathrm{H})$, as we have for $\left(T_{\gamma}\right)$. Such a criterion would be a great help to answer the two previous questions because condition $(\mathrm{H})$ is easy to understand but hard to verify.

Finally, notice that Corollary 1 has a version for velocities instead of asymptotic directions. More precisely, $P_{0}$-almost surely, the limit of $X_{n} / n$ belongs to a set $\left\{S_{1}, S_{2}\right\}$ such that if $S_{1} \neq 0$ then $S_{2}=\lambda S_{1}$ for some $\lambda \leqslant 0$ (Theorem 1.1 in [1]). However none of these two results can be deduced from the other one.

The proofs of the results will be given in the second part of this paper. We finish this section with some notation which will be useful in the proofs. Denote by $\theta_{n}$ the time shift ( $n$ natural number is the argument) and by $t_{x}$ the spatial shift ( $x$ in $\mathbb{Z}^{d}$ is the argument). For any fixed $\ell$ in $\mathbb{R}_{*}^{d}$, we let $T_{u}$ be the hitting time of the open half-space $\left\{x \in \mathbb{Z}^{d}, x \cdot \ell>u\right\}$

$$
T_{u}=\inf \left\{n>0, X_{n} \cdot \ell>u\right\},
$$

and $D^{\ell}$ the return time of the walk behind the starting point

$$
D^{\ell}=\inf \left\{n>0, X_{n} \cdot \ell \leqslant X_{0} \cdot \ell\right\} .
$$

Notice that these two definitions are quite different from those used in [8].

We complete $\ell$ into an orthogonal basis $\left(e_{2}, \ldots, e_{d}\right)$, such that for every $i$ in $\llbracket 2, d \rrbracket,\left|e_{i}\right|=1$.

For all $i \in \llbracket 2, d \rrbracket$ we define the following two vectors:

$$
\ell_{i}^{\prime}(\alpha)=\ell+\alpha e_{i} \quad \text { and } \quad \ell_{-i}^{\prime}(\alpha)=\ell-\alpha e_{i} .
$$

For all positive real $\alpha$ we can define the convex cone $C(\alpha)$ by

$$
C(\alpha)=\bigcap_{i=2}^{d}\left\{x \in \mathbb{Z}^{d}, x \cdot \ell_{i}^{\prime}(\alpha) \geqslant 0 \text { and } x \cdot \ell_{-i}^{\prime}(\alpha) \geqslant 0\right\} .
$$

We also define the exit time $D_{\alpha}^{\ell}$ of the cone $C(\alpha)$, shifted at the starting point of the walk,

$$
D_{\alpha}^{\ell}=\inf \left\{n \geqslant 0, \exists i \in \llbracket 2, d \rrbracket, X_{n} \cdot \ell_{i}^{\prime}(\alpha)<X_{0} \cdot \ell_{i}^{\prime}(\alpha) \text { or } X_{n} \cdot \ell_{-i}^{\prime}(\alpha)<X_{0} \cdot \ell_{-i}^{\prime}(\alpha)\right\} .
$$

Notice that under $P_{0}, D_{\alpha}^{\ell}$ can also be defined in the following way,

$$
P_{0} \text {-a.s., } \quad D_{\alpha}^{\ell}=\inf \left\{n \geqslant 0, X_{n} \notin C(\alpha)\right\} \text {. }
$$




\section{Proofs}

Proof of Theorem 1. The first step of the proof is the following lemma, where it is proved that under $(\mathrm{H})$ the walk has a positive probability never to exit a cone $C(\alpha)$ for $\alpha$ small enough.

Lemma 1. Let $\ell$ be a vector in $\mathbb{R}^{d}$ satisfying $(\mathrm{H})$ then, for any choice of an orthogonal basis $\left(\ell, e_{2}, \ldots, e_{d}\right)$ with $\left|e_{i}\right|=1$ for any $i$ in $\llbracket 2, d \rrbracket$, there exist some $\alpha_{0}>0$ such that,

$$
\forall \alpha \leqslant \alpha_{0} \quad P_{0}\left(D_{\alpha}^{\ell}=\infty\right)>0 .
$$

Proof. Fix a basis satisfying the assumption of the lemma. We will first show that there exists a random variable $\alpha_{1}>0$ such that

$$
P_{0}\left(D_{\alpha_{1}}^{\ell}=\infty \mid D^{\ell}=\infty\right)=1 .
$$

Since $\mathcal{V}$ is an open set, there exist some $\alpha_{2}>0$ such that for every $i \in \llbracket 2, d \rrbracket$ :

$$
\ell_{i}^{\prime}\left(\alpha_{2}\right) \in \mathcal{V} \quad \text { and } \quad \ell_{-i}^{\prime}\left(\alpha_{2}\right) \in \mathcal{V} .
$$

For these $(2 d-2)$ directions, we use the renewal structure described in Section 1 of [8]. The choice of the parameter $a$ in this structure has no importance and can be done arbitrarily. Remember that, for any fixed direction $\ell$, the first renewal time $\tau^{\ell}$ of [8] is the first time the walk reaches a new record in direction $\ell$, and later never backtracks.

Remark 1. In [8], as in further references, uniform ellipticity is assumed. When we quote these articles, we have verified that this stronger assumption is not necessary or can be relaxed as in [11].

Using (H) we obtain that for each $i \in \llbracket 2, d \rrbracket$,

$$
P_{0}\left(A_{\ell_{i}^{\prime}\left(\alpha_{2}\right)}\right)=P_{0}\left(A_{\ell_{-i}^{\prime}\left(\alpha_{2}\right)}\right)=1 \text {. }
$$

From Proposition 1.2 in [8]:

$$
\tau^{\ell_{2}^{\prime}\left(\alpha_{2}\right)} \vee \cdots \vee \tau^{\ell_{d}^{\prime}\left(\alpha_{2}\right)} \vee \tau_{-2}^{\ell_{-2}^{\prime}\left(\alpha_{2}\right)} \vee \cdots \vee \tau^{\ell_{-d}^{\prime}\left(\alpha_{2}\right)}<\infty \quad P_{0} \text {-a.s. }
$$

Using a proof very close to Proposition 1.2 in [8] (see also Theorem 3 in [4]) we obtain $P_{0}\left(D^{\ell}=\infty\right)>0$ and so:

$$
\tau^{\ell_{2}^{\prime}\left(\alpha_{2}\right)} \vee \cdots \vee \tau^{\ell_{d}^{\prime}\left(\alpha_{2}\right)} \vee \tau_{-2}^{\ell_{-2}^{\prime}\left(\alpha_{2}\right)} \vee \cdots \vee \tau_{-d}^{\ell^{\prime}\left(\alpha_{2}\right)}<\infty \quad P_{0}\left(\cdot \mid D^{\ell}=\infty\right) \text {-a.s. }
$$

We now define the following variables:

$$
\begin{aligned}
& N=\inf \left\{n_{0} \geqslant 1, \forall n \geqslant n_{0}, X_{n} \in C\left(\alpha_{2}\right)\right\} \quad(\inf \emptyset=+\infty), \\
& C=\inf _{1 \leqslant n \leqslant N} X_{n} \cdot \ell, \\
& M=\sup _{1 \leqslant n \leqslant N} \sum_{i=2}^{d}\left|X_{n} \cdot e_{i}\right|^{2} .
\end{aligned}
$$

From (4), it is clear that:

$$
\begin{aligned}
& P_{0}\left(\cdot \mid D^{\ell}=\infty\right) \text {-a.s., } \quad N<\infty, \\
& C>0 \text { and } M<\infty .
\end{aligned}
$$

We now define $\alpha_{1}=\frac{C}{\sqrt{M}} \wedge \alpha_{2}$ (notice that $\alpha_{1}$ is random), using Cauchy-Schwarz inequality for $n \leqslant N$ and the definition of $N$ and $C(\alpha)$ for $n \geqslant N$, we obtain:

$$
\begin{aligned}
& P_{0}\left(\cdot \mid D^{\ell}=\infty\right) \text {-a.s., } \forall i \in \llbracket 2, d \rrbracket \forall n \geqslant 0, \quad X_{n} \cdot \ell_{i}^{\prime}\left(\alpha_{1}\right)=X_{n} \cdot \ell+\alpha_{1}\left(X_{n} \cdot e_{i}\right) \geqslant 0, \\
& \quad \text { and } \quad X_{n} \cdot \ell_{-i}^{\prime}\left(\alpha_{1}\right)=X_{n} \cdot \ell-\alpha_{1}\left(X_{n} \cdot e_{i}\right) \geqslant 0
\end{aligned}
$$

which ends the proof of (3). 
It is clear that

$$
\alpha<\alpha^{\prime} \text { implies } C\left(\alpha^{\prime}\right) \subset C(\alpha),
$$

and so

$$
\lim _{\alpha \rightarrow 0} P_{0}\left(\left\{D_{\alpha}^{\ell}=\infty\right\}\right)=P_{0}\left(\bigcup_{\alpha>0}\left\{D_{\alpha}^{\ell}=\infty\right\}\right) .
$$

From (3), we have

$$
\bigcup_{\alpha>0}\left\{D_{\alpha}^{\ell}=\infty\right\} \stackrel{P_{0} \text {-a.s. }}{=}\left\{D^{\ell}=\infty\right\} \text {. }
$$

Since $P_{0}\left(D^{\ell}=\infty\right)>0$, this concludes the proof of Lemma 1 .

We will now construct a renewal structure in the same spirit as in [8] or [2]. The idea is to define a time where the walk reaches a new record in the direction $\ell$ and never goes out of a cone (also oriented in direction $\ell$ ) after. In [8], the walk moves from one slab to the next one, here, as in [2] or [3], the walk will move from one cone to the next one. From Lemma 1, we know that we can choose $\alpha$ small enough so that

$$
P_{0}\left(D_{\alpha}^{\ell}=\infty\right)>0 .
$$

We define now the two stopping time sequences $\left(S_{k}\right)_{k} \geqslant 0$ and $\left(R_{k}\right)_{k} \geqslant 0$, and the sequence of successive maxima $\left(M_{k}\right)_{k} \geqslant 0$

$$
S_{0}=\inf \left\{n \geqslant 0, X_{n} \cdot \ell>X_{0} \cdot \ell\right\}, \quad R_{0}=D_{\alpha}^{\ell} \circ \theta_{S_{0}}+S_{0}, \quad M_{0}=\sup \left\{\ell \cdot X_{n}, 0 \leqslant n \leqslant R_{0}\right\} .
$$

And for all $k \geqslant 0$ :

$$
\begin{aligned}
& S_{k+1}=T_{M_{k}}, \quad R_{k+1}=D_{\alpha}^{\ell} \circ \theta_{S_{k+1}}+S_{k+1}, \quad M_{k+1}=\sup \left\{\ell \cdot X_{n}, 0 \leqslant n \leqslant R_{k+1}\right\}, \\
& K=\inf \left\{k \geqslant 0, S_{k}<\infty, R_{k}=\infty\right\} .
\end{aligned}
$$

On the set $K<\infty$, we also define:

$$
\tau_{1}=S_{K}
$$

The random time $\tau_{1}$ is called the first cone renewal time, and will not be confused with $\tau^{\ell}$ introduced above. Under assumption $(\mathrm{H})$,

$$
S_{0} \leqslant R_{0}<S_{1} \leqslant R_{1}<\cdots<S_{n} \leqslant R_{n}<\cdots \leqslant \infty .
$$

Proposition 2. Under assumption $(\mathrm{H})$,

$$
\text { P0-a.s. } \quad K<\infty .
$$

Proof. For all $k \geqslant 1$,

$$
\begin{aligned}
P_{0}\left(R_{k}<\infty\right) & =\mathbb{E}\left[E_{0, \omega}\left[S_{k}<\infty, D_{\alpha}^{\ell} \circ \theta_{S_{k}}<\infty\right]\right] \\
& =\sum_{x \in \mathbb{Z}^{d}} \mathbb{E}\left[E_{0, \omega}\left[S_{k}<\infty, X_{S_{k}}=x, D_{\alpha}^{\ell} \circ \theta_{S_{k}}<\infty\right]\right] .
\end{aligned}
$$

Using Markov property we obtain,

$$
P_{0}\left(R_{k}<\infty\right)=\sum_{x \in \mathbb{Z}^{d}} \mathbb{E}\left[E_{0, \omega}\left[S_{k}<\infty, X_{S_{k}}=x\right] E_{x, \omega}\left[D_{\alpha}^{\ell}<\infty\right]\right]
$$

For every $x$ in $\mathbb{Z}^{d}$, the variables $E_{0, \omega}\left[S_{k}<\infty, X_{S_{k}}=x\right]$ and $E_{x, \omega}\left[D_{\alpha}^{\ell}<\infty\right]$ are respectively $\sigma\left\{\omega_{y}(\cdot), \ell \cdot y<\ell \cdot x\right\}$ and $\sigma\left\{\omega_{y}(\cdot), y \in t_{x} \circ C(\alpha)\right\}$ measurable. As this two $\sigma$-fields are independent, 


$$
\begin{aligned}
P_{0}\left(R_{k}<\infty\right) & =\sum_{x \in \mathbb{Z}^{d}} E_{0}\left[S_{k}<\infty, X_{S_{k}}=x\right] E_{x}\left[D_{\alpha}^{\ell}<\infty\right] \\
& =P_{0}\left(S_{k}<\infty\right) P_{0}\left(D_{\alpha}^{\ell}<\infty\right) \\
& =P_{0}\left(R_{k-1}<\infty\right) P_{0}\left(D_{\alpha}^{\ell}<\infty\right) .
\end{aligned}
$$

By induction, we obtain,

$$
P_{0}\left(R_{k}<\infty\right)=P_{0}\left(D_{\alpha}^{\ell}<\infty\right)^{k+1} .
$$

In view of (5), this concludes the proof.

We now define a sequence of renewal time $\left(\tau_{k}\right)_{k} \geqslant 1$ by the following recursive relation:

$$
\tau_{k+1}=\tau_{1}(X .)+\tau_{k}\left(X_{\tau_{1}+.}-X_{\tau_{1}}\right) .
$$

Using Proposition 2, we have:

$$
\forall k \geqslant 0, \quad \tau_{k}<\infty .
$$

Proposition 3. Under assumption $(\mathrm{H})$,

$$
\left(\left(X_{\tau_{1} \wedge .}\right), \tau_{1}\right),\left(\left(X_{\left(\tau_{1}+\cdot\right) \wedge \tau_{2}}-X_{\tau_{1}}\right), \tau_{2}-\tau_{1}\right), \ldots,\left(\left(X_{\left(\tau_{k}+\cdot\right) \wedge \tau_{k+1}}-X_{\tau_{k}}\right), \tau_{k+1}-\tau_{k}\right)
$$

are independent variables under $P_{0}$ and for $k \geqslant 1,\left(\left(X_{\left(\tau_{k}+\cdot\right) \wedge \tau_{k+1}}-X_{\tau_{k}}\right), \tau_{k+1}-\tau_{k}\right)$ are distributed like $\left(\left(X_{\left.\tau_{1} \wedge .\right)}\right), \tau_{1}\right)$ under $P_{0}\left(\cdot \mid D_{\alpha}^{\ell}=\infty\right)$.

The proof is similar to that of Corollary 1.5 in [8] and will not be repeated here.

For the classical renewal structure, Zerner proved that $E_{0}\left[X_{\tau_{1}} \cdot \ell\right]$ is finite and computes its value. We provide here the same result but for a renewal structure with cones.

Fix a direction $\ell$ with integer coordinates $\left(a_{1}, \ldots, a_{d}\right)$ such that their greatest common divisor, $\operatorname{gcd}\left(a_{1}, \ldots, a_{d}\right)=1$. Assume that $(\mathrm{H})$ is satisfied for $\ell$. Complete $\ell$ in an orthogonal basis $\left(\ell, e_{2}, \ldots, e_{d}\right)$ such that for every $i$ in $\llbracket 2, d \rrbracket,\left|e_{i}\right|=1$. By Lemma 1 , we can choose $\alpha$ small enough so that $P_{0}\left(D_{\alpha}^{\ell}=\infty\right)>0$ and construct the associated renewal structure that is described above.

Lemma 2. Under assumption $(\mathrm{H})$,

$$
E_{0}\left[X_{\tau_{1}} \cdot \ell \mid D_{\alpha}^{\ell}=\infty\right]=\frac{1}{P_{0}\left(D_{\alpha}^{\ell}=\infty\right)} .
$$

Proof. This proof follows an unpublished argument of M. Zerner but can be found in Lemma 3.2.5 p. 265 of [10]. Since $\operatorname{gcd}\left(a_{1}, \ldots, a_{d}\right)=1$, we have $\left\{x \cdot \ell, x \in \mathbb{Z}^{d}\right\}=\mathbb{Z}$. For all $i>0$,

$$
\begin{aligned}
P_{0}\left(\left\{\exists k \geqslant 1, X_{\tau_{k}} \cdot \ell=i\right\}\right) & =\sum_{\left\{x \in \mathbb{Z}^{d}, x \cdot \ell=i\right\}} \mathbb{E}\left[E_{0, \omega}\left[\left\{X_{T_{i-1}}=x, D_{\alpha}^{\ell} \circ \theta_{T_{i-1}}=\infty\right\}\right]\right] \\
& =\sum_{\left\{x \in \mathbb{Z}^{d}, x \cdot \ell=i\right\}} \mathbb{E}\left[E_{0, \omega}\left[X_{T_{i-1}}=x\right] E_{x, \omega}\left[D_{\alpha}^{\ell}=\infty\right]\right] \\
& =P_{0}\left(D_{\alpha}^{\ell}=\infty\right) .
\end{aligned}
$$

We used the strong Markov property in (7).

In (8), we notice that $E_{0, \omega}\left(X_{T_{i-1}}=x\right)$ is $\sigma\left\{\omega_{y}(\cdot), \ell \cdot y<\ell \cdot x\right\}$ measurable and $E_{x, \omega}\left(D_{\alpha}^{\ell}=\infty\right)$ is $\sigma\left\{\omega_{y}(\cdot), y \in\right.$ $\left.t_{x} \circ C(\alpha)\right\}$ measurable and that those two $\sigma$-fields are independent. We will now compute the same value in another way. 


$$
\begin{aligned}
\lim _{i \rightarrow \infty} P_{0}\left(\left\{\exists k \geqslant 1, X_{\tau_{k}} \cdot \ell=i\right\}\right) & =\lim _{i \rightarrow \infty} P_{0}\left(\left\{\exists k \geqslant 2, X_{\tau_{k}} \cdot \ell=i\right\}\right) \\
& =\lim _{i \rightarrow \infty} \sum_{n \geqslant 1} P_{0}\left(\left\{\exists k \geqslant 2,\left(X_{\tau_{k}}-X_{\tau_{1}}\right) \cdot \ell=i-n, X_{\tau_{1}} \cdot \ell=n\right\}\right) \\
& =\lim _{i \rightarrow \infty} \sum_{n \geqslant 1} P_{0}\left(\left\{\exists k \geqslant 2,\left(X_{\tau_{k}}-X_{\tau_{1}}\right) \cdot \ell=i-n\right\}\right) P_{0}\left(X_{\tau_{1}} \cdot \ell=n\right) .
\end{aligned}
$$

Notice also that the first equality is true because $P_{0}\left(X_{\tau_{1}} \cdot \ell>i\right) \rightarrow 0(i \rightarrow \infty)$. Using now the renewal theorem (Corollary 10.2 p. 76 in [9]) we obtain

$$
\lim _{i \rightarrow \infty} P_{0}\left(\left\{\exists k \geqslant 2,\left(X_{\tau_{k}}-X_{\tau_{1}}\right) \cdot \ell=i-n\right\}\right)=\frac{1}{E_{0}\left[\left(X_{\tau_{2}}-X_{\tau_{1}}\right) \cdot \ell\right]} .
$$

The dominated convergence theorem leads to

$$
\lim _{i \rightarrow \infty} P_{0}\left(\left\{\exists k \geqslant 1, X_{\tau_{k}} \cdot \ell=i\right\}\right)=\frac{1}{E_{0}\left[\left(X_{\tau_{2}}-X_{\tau_{1}}\right) \cdot \ell\right]} .
$$

Comparing this result with (8), we easily obtain Lemma 2.

We have now all the tools to prove Theorem 1. We will first use the two lemmas to prove that (i) implies (ii).

We choose $\ell$ with rational coordinates in the open set $\mathcal{O}$. It is clear that $\ell$ satisfies assumption $(\mathrm{H})$. Actually, we can also assume, without loss of generality, that $\ell$ has integer coordinates and that their greatest common divisor is 1 . Indeed, there is $\lambda$ rational such that $\lambda \ell$ has integer coordinates with greatest common divisor equal to 1 , and of course, $\lambda \ell$ also satisfies $(\mathrm{H})$.

We complete $\ell$ into an orthogonal basis $\left(e_{2}, \ldots, e_{d}\right)$, such that for every $i$ in $\llbracket 2, d \rrbracket,\left|e_{i}\right|=1$. Using Lemma 1 , we choose $\alpha$ small enough so that $P_{0}\left(D_{\alpha}^{\ell}=\infty\right)>0$. We can now use the renewal structure with cones and we have from Lemma 2 that:

$$
E_{0}\left[X_{\tau_{1}} \cdot \ell \mid D_{\alpha}^{\ell}=\infty\right]=\frac{1}{P_{0}\left(D_{\alpha}^{\ell}=\infty\right)}<\infty
$$

From the definition of the cone renewal structure, there is some constant $c(\alpha)>0$ such that, $P_{0}\left(\cdot \mid D_{\alpha}^{\ell}=\infty\right)$-a.s., for any time $n$,

$$
\left|X_{n}\right| \leqslant c(\alpha) X_{n} \cdot \ell,
$$

and so using Lemma 2 ,

$$
E_{0}\left[\left|X_{\tau_{1}}\right| \mid D_{\alpha}^{\ell}=\infty\right]<\infty .
$$

We can now apply the law of large numbers, and obtain

$$
\frac{X_{\tau_{k}}}{k} \underset{n \rightarrow \infty}{\longrightarrow} E_{0}\left[X_{\tau_{1}} \mid D_{\alpha}^{\ell}=\infty\right] \quad P_{0} \text {-a.s. }
$$

As $\left|E_{0}\left[X_{\tau_{1}} \mid D_{\alpha}^{\ell}=\infty\right]\right|>0$,

$$
\frac{X_{\tau_{k}}}{\left|X_{\tau_{k}}\right|} \underset{n \rightarrow \infty}{\longrightarrow} \frac{E_{0}\left[X_{\tau_{1}} \mid D_{\alpha}^{\ell}=\infty\right]}{\left|E_{0}\left[X_{\tau_{1}} \mid D_{\alpha}^{\ell}=\infty\right]\right|} \stackrel{\text { (def) }}{=} v \quad P_{0} \text {-a.s. }
$$

To complete the proof, we have to control the behavior of the walk between the renewal times. For each natural $n$, we introduce the index $k(n)$ such that,

$$
\tau_{k(n)} \leqslant n<\tau_{k(n)+1} .
$$

Recall that if $\left(Z_{n}\right)$ is an i.i.d. sequence of variables with finite expectation, Borel-Cantelli Lemma assures that $Z_{n} / n$ converges almost surely to 0 . From (9), Lemma 2 and Proposition 3, the sequence $\left(\sup _{n}\left|X_{\tau_{k}+n \wedge \tau_{k+1}}-X_{\tau_{k}}\right|\right)_{k} \geqslant 1$ is i.i.d. with finite expectation and we can apply the previous remark to obtain:

$$
\frac{\sup _{n}\left|X_{\tau_{k}+n \wedge \tau_{k+1}}-X_{\tau_{k}}\right|}{k} \underset{k \rightarrow \infty}{\longrightarrow} 0 \quad P_{0} \text {-a.s. }
$$


Using Eqs. (12) and (13), we study the convergence in (ii),

$$
\frac{X_{n}}{\left|X_{n}\right|}=\frac{X_{n}-X_{\tau_{k(n)}}}{\left|X_{n}\right|}+\frac{X_{\tau_{k(n)}}}{k(n)} \frac{k(n)}{\left|X_{n}\right|} .
$$

By Proposition 2 and (6),

$$
k(n) \underset{n \rightarrow \infty}{\longrightarrow} \infty \quad P_{0} \text {-a.s. }
$$

As $\left|X_{n}\right| \geqslant k(n)$, (13) leads to:

$$
\frac{X_{n}-X_{\tau_{k(n)}}}{\left|X_{n}\right|} \underset{n \rightarrow \infty}{\longrightarrow} 0 \quad P_{0} \text {-a.s. }
$$

To control the second term in (14), we simply write

$$
\frac{\left|X_{\tau_{k(n)}}\right|}{k(n)}-\frac{\left|X_{n}-X_{\tau_{k(n)}}\right|}{k(n)} \leqslant \frac{\left|X_{n}\right|}{k(n)} \leqslant \frac{\left|X_{\tau_{k(n)}}\right|}{k(n)}+\frac{\left|X_{n}-X_{\tau_{k(n)}}\right|}{k(n)} .
$$

Using (11) and (13), we obtain,

$$
\frac{\left|X_{n}\right|}{k(n)} \underset{n \rightarrow \infty}{\longrightarrow}\left|E_{0}\left[X_{\tau_{1}} \cdot \ell \mid D_{\alpha}^{\ell}=\infty\right]\right| \quad P_{0} \text {-a.s. }
$$

We finally obtain the desired convergence:

$$
\frac{X_{n}}{\left|X_{n}\right|} \underset{n \rightarrow \infty}{\longrightarrow} v=\frac{E_{0}\left[X_{\tau_{1}} \mid D_{\alpha}^{\ell}=\infty\right]}{\left|E_{0}\left[X_{\tau_{1}} \mid D_{\alpha}^{\ell}=\infty\right]\right|} \quad P_{0} \text {-a.s. }
$$

The end of the proof of Theorem 1 is easy: it is obvious that (iii) implies (i) and so we just have to show that (ii) implies (iii).

Let $\ell$ be a direction such that $\ell \cdot v>0$. It is known since [8] (Lemma 1.1) that $P_{0}\left(A_{\ell} \cup A_{-\ell}\right)$ follows a $0-1$ law under assumption of uniform ellipticity, but we use here Proposition 3 in [11] where the same result is proved under the weaker assumption of strict ellipticity.

If $P_{0}\left(A_{\ell} \cup A_{-\ell}\right)=0$, it is known that the walk under $P_{0}$ oscillates,

$$
\limsup _{n \rightarrow \infty} X_{n} \cdot \ell=-\liminf _{n \rightarrow \infty} X_{n} \cdot \ell=+\infty \quad P_{0} \text {-a.s. }
$$

This is not possible in view of (ii) and so $P_{0}\left(A_{\ell} \cup A_{-\ell}\right)=1$.

But because of (ii), $P_{0}\left(A_{-\ell}\right)=0$, and we can conclude

$$
P_{0}\left(A_{\ell}\right)=1 .
$$

Remark 2. From the proof of Theorem 1, we know that if a walk has an asymptotic direction, we can construct a renewal structure with cones and $E\left[X_{\tau_{1}} \mid D_{\alpha}^{\ell}=\infty\right]$ is finite. We can then easily derive a law of large numbers, namely

$$
\frac{X_{n}}{n} \underset{n \rightarrow \infty}{\longrightarrow} \frac{E_{0}\left[X_{\tau_{1}} \mid D_{\alpha}^{\ell}=\infty\right]}{E_{0}\left[\tau_{1} \mid D_{\alpha}^{\ell}=\infty\right]} \stackrel{\text { (def) }}{=} \mu \quad P_{0} \text {-a.s. }
$$

However this limit can be zero (if and only if $E_{0}\left[\tau_{1} \mid D_{\alpha}^{\ell}=\infty\right]=+\infty$ ) and, in this case, the asymptotic direction is an interesting information about the walk's behavior.

Remark 3. If a walk admits an asymptotic direction, we know that the walk is transient in any direction $\ell$ satisfying $\ell \cdot v>0$. For any such $\ell$, we can consider a slab renewal structure defined as the cone renewal structure except that in the definitions of $\left(R_{k}\right)_{k} \geqslant 0,\left(S_{k}\right)_{k} \geqslant 0$ and $\left(M_{k}\right)_{k} \geqslant 0, D_{\alpha}^{\ell}$ is replaced by $\tilde{D}^{\ell}=\inf \left\{n>0, X_{n} \cdot \ell<X_{0} \cdot \ell\right\}$ (this construction is very similar to that of [8]). For any $k \geqslant 1$, $\tau_{k}^{\ell}$ will denote the $k$-th slab renewal time. The variables $\left(X_{\tau_{k+1}^{\ell}}-X_{\tau_{k}^{\ell}}\right)_{k} \geqslant 1$ are i.i.d., and the purpose of this remark is to show that the expectation of their norm, $E_{0}\left[\left|X_{\tau_{1}^{\ell}}\right| \mid \tilde{D}^{\ell}=\infty\right]$ is finite. 
From Corollary 3 in [5], it is enough to prove that $\left(X_{\tau_{k}^{\ell}} / k\right)_{k \geqslant 1}$ is bounded $P_{0}$-almost surely. ${ }^{2}$ For any $k \geqslant 1$, we introduce

$$
J(k)=\sup \left\{j \geqslant 1, \tau_{j} \leqslant \tau_{k}^{\ell}\right\} \quad(\sup \emptyset=0) .
$$

As $P_{0}$-almost surely $\left(\tau_{k}^{\ell}\right)_{k \geqslant 1}$ is increasing, we have

$$
\lim _{k \rightarrow \infty} J(k)=\infty \quad P_{0} \text {-a.s. }
$$

Notice that a cone renewal time is also a slab renewal time and as a consequence

$$
J(k) \leqslant k \quad P_{0} \text {-a.s. }
$$

$P_{0}$-a.s. for large $k$ so that $J(k) \geqslant 1$ :

$$
\frac{X_{\tau_{k}^{\ell}}}{k}=\frac{X_{\tau_{k}^{\ell}}-X_{\tau_{J(k)}}}{k}+\frac{X_{\tau_{J(k)}}}{k} .
$$

The norm of the first term is bounded by $c(\alpha)\left|X_{\tau_{J(k)+1}} \cdot \ell-X_{\tau_{J(k)}} \cdot \ell\right| / J(k)$. From the same argument as in (13), $c(\alpha)\left|X_{\tau_{j+1}} \cdot \ell-X_{\tau_{j}} \cdot \ell\right| / j$ converges $P_{0}$-almost surely to 0 , and using (16), we obtain the $P_{0}$-almost surely convergence of the first term to 0 . Rewriting the second term

$$
\frac{X_{\tau_{J(k)}}}{k}=\frac{X_{\tau_{J(k)}}}{J(k)} \frac{J(k)}{k},
$$

from (17), (16) and (11) we obtain that the second term is almost surely bounded as $k$ goes to infinity.

Proof of Proposition 1. Suppose that the proposition is false and call $v$ and $v^{\prime}$ two vectors of $S^{d-1}$ different and non-opposite such that $P_{0}\left(B_{v}\right) P_{0}\left(B_{v^{\prime}}\right)>0$, then we will show that

$\exists v_{0}$ such that $P_{0}\left(B_{v_{0}} \mid B_{v} \cup B_{v^{\prime}}\right)=1$,

what establishes, of course, a contradiction.

First, notice that for $v \in S^{d-1}$ with $P_{0}\left(B_{v}\right)>0$ we have,

$$
\forall \ell \in \mathbb{R}^{d} \text { such that } \ell \cdot v>0, \quad P_{0}\left(A_{\ell} \mid B_{v}\right)=1 .
$$

Indeed, from the 0-1 law $P_{0}\left(A_{\ell} \cup A_{-\ell}\right)=1$ (just notice that the walk does not oscillate along direction $\ell$ on $B_{\nu}$, event of positive probability), but since $B_{v} \subset\left\{\exists N\right.$ s.t. $\left.\forall n \geqslant N, X_{n} \cdot \ell>0\right\}$, we have $B_{v} \subset A_{\ell}, P_{0}$-almost surely, which implies (19).

The set $\mathcal{V}=\left\{\ell \in \mathbb{R}^{d}, \ell \cdot v>0\right\} \cap\left\{\ell \in \mathbb{R}^{d}, \ell \cdot v^{\prime}>0\right\}$ is non-empty and open and from (19) it has the property,

$$
\forall \ell \in \mathcal{V}, \quad P_{0}\left(A_{\ell} \mid B_{v} \cup B_{v^{\prime}}\right)=1 .
$$

From now on, we fix $\ell_{0}$ in $\mathcal{V}$. The property $\left(\mathrm{H}^{\prime}\right)$ is similar to assumption $(\mathrm{H})$ and the proof of (18) will be adapted from that of Theorem 1. In fact, we will use the cone renewal structure on $A_{\ell_{0}}$ and show

$$
\exists v_{0} \text { s.t. } P_{0}\left(B_{\nu_{0}} \mid A_{\ell_{0}}\right)=1 \text {, }
$$

which is stronger that (18). Before the proof, notice that if $P_{0}\left(B_{v} \cup B_{v^{\prime}}\right)=1$, we can easily conclude using Theorem 1, but the proof is not that obvious if $P_{0}\left(B_{v} \cup B_{v^{\prime}}\right)<1$.

In order to construct a renewal structure with cones on the event $A_{\ell_{0}}$ of positive probability, we have to show that there exists $\alpha>0$ such that $P_{0}\left(D_{\alpha}^{\ell_{0}}=\infty\right)>0$. We will use a proof very close to the one of Lemma 1 except that we will work on the event $\left\{B_{v} \cup B_{v^{\prime}}\right\}$ and show the stronger result $P_{0}\left(D_{\alpha}^{\ell_{0}}=\infty, B_{v} \cup B_{v^{\prime}}\right)>0$. Our first step will be to prove that

$$
P_{0}\left(D^{\ell_{0}}=\infty, B_{v} \cup B_{v^{\prime}}\right)>0 .
$$

\footnotetext{
2 The result in [5] is for $d=1$. Considering all the coordinates separately, the claim in arbitrary dimension follows.
} 
We argue by contradiction and assume the left-hand side of (21) is zero. By translation-invariance, it holds $P_{x}\left(D^{\ell_{0}}=\infty, B_{v} \cup B_{v^{\prime}}\right)=0$ for any $x$ in $\mathbb{Z}^{d}$, which means

$$
\forall x \in \mathbb{Z}^{d}, \mathbb{P} \text {-a.s., } \quad P_{x, \omega}\left(D^{\ell_{0}}=\infty, B_{v} \cup B_{v^{\prime}}\right)=0,
$$

and also

$$
\text { P-a.s., } \forall x \in \mathbb{Z}^{d}, \quad P_{x, \omega}\left(D^{\ell_{0}}=\infty, B_{v} \cup B_{v^{\prime}}\right)=0 .
$$

We denote by $\left(D^{\ell_{0}}\right)^{n}$ the $n$-th backtrack time of the walk, defined by the following recursive relation

$$
\begin{aligned}
& \left(D^{\ell_{0}}\right)^{1}=D^{\ell_{0}}, \\
& \left(D^{\ell_{0}}\right)^{n}=D^{\ell_{0}} \circ \theta_{\left(D^{\ell_{0}}\right)^{n-1}} 1_{\left\{\left(D^{\ell_{0}}\right)^{n-1}<\infty\right\}}+\infty 1_{\left.\left\{D^{\ell_{0}}\right)^{n-1}=\infty\right\}}, \quad \forall n \geqslant 2 .
\end{aligned}
$$

Notice that for any $n \geqslant 1$,

$$
\begin{aligned}
& \left\{\left(D^{\ell_{0}}\right)^{n}<\infty,\left(D^{\ell_{0}}\right)^{n+1}=\infty, B_{v} \cup B_{v^{\prime}}\right\} \\
& \quad=\bigcup_{x \in \mathbb{Z}^{d}} \bigcup_{m \geqslant 1}\left\{\left(D^{\ell_{0}}\right)^{n}<\infty,\left(D^{\ell_{0}}\right)^{n}=m, X_{m}=x\right\} \cap\left\{D^{\ell_{0}} \circ \theta_{m}=\infty, \lim _{k \rightarrow \infty} \frac{X_{k}-x}{\left|X_{k}-x\right|} \in\left\{v, v^{\prime}\right\}\right\} .
\end{aligned}
$$

We can then use the Markov property to show that $\mathbb{P}$-a.s., for any $n \geqslant 1$,

$$
\begin{aligned}
& P_{0, \omega}\left(\left(D^{\ell_{0}}\right)^{n}<\infty,\left(D^{\ell_{0}}\right)^{n+1}=\infty, B_{v} \cup B_{v^{\prime}}\right) \\
& \quad=\sum_{x \in \mathbb{Z}^{d}} P_{0, \omega}\left(\left(D^{\ell_{0}}\right)^{n}<\infty, X_{\left(D^{\left.\ell_{0}\right)^{n}}\right.}=x\right) P_{x, \omega}\left(D^{\ell_{0}}=\infty, B_{v} \cup B_{v^{\prime}}\right) .
\end{aligned}
$$

We finally obtain $P_{0}\left(\left(D^{\ell}\right)^{n}<\infty, \forall n \geqslant 1 \mid B_{v} \cup B_{v^{\prime}}\right)=1$. This establishes a contradiction with $\left(\mathrm{H}^{\prime}\right)$ and concludes the proof of (21). It is possible to choose $\alpha>0$ such that for every $i \in \llbracket 2, d \rrbracket$ :

$$
\ell_{i}^{\prime}(\alpha) \in \mathcal{V} \quad \text { and } \quad \ell_{-i}^{\prime}(\alpha) \in \mathcal{V},
$$

in the notations of (1) with $\ell=\ell_{0}$. From (19) and Proposition 1.2 in [8], it is clear that almost surely on $\left\{B_{v} \cup B_{v^{\prime}}\right\} \cap$ $\left\{D^{\ell_{0}}=\infty\right\}$,

$$
\tau^{\ell_{2}^{\prime}(\alpha)} \vee \cdots \vee \tau^{\ell_{d}^{\prime}(\alpha)} \vee \tau^{\ell_{-2}^{\prime}(\alpha)} \vee \cdots \vee \tau^{\ell_{-d}^{\prime}(\alpha)}<\infty
$$

We can then follow the end of the proof of Lemma 1 and we obtain:

$$
\bigcup_{\alpha>0}\left\{D_{\alpha}^{\ell_{0}}=\infty, B_{v} \cup B_{v^{\prime}}\right\} \stackrel{P_{0} \text {-a.s. }}{=}\left\{D^{\ell_{0}}=\infty, B_{v} \cup B_{v^{\prime}}\right\},
$$

and hence we can fix $\alpha>0$ such that,

$$
P_{0}\left(D_{\alpha}^{\ell_{0}}=\infty, B_{v} \cup B_{v^{\prime}}\right)>0 .
$$

We will now show that on $A_{\ell_{0}}$ (this is much easier than on $B_{v} \cup B_{v^{\prime}}$ ), the walk admits almost surely a unique asymptotic direction. We use the same renewal structure with cones as in the proof of Theorem 1. Following the proof of Proposition 1.2 in [8], we obtain

Proposition 4. Under assumption $\left(\mathrm{H}^{\prime}\right)$,

$$
P_{0} \text {-a.s., } \quad A_{\ell_{0}}=\{K<\infty\}=\left\{\tau_{1}<\infty\right\} .
$$

We also adopt the notation $Q_{0}$ to denote the probability measure $P_{0}\left(\cdot \mid A_{\ell_{0}}\right)$. The proof of Corollary 1.5 in [8] leads to,

Proposition 5. Under assumption $\left(\mathrm{H}^{\prime}\right)$,

$$
\left(\left(X_{\left.\tau_{1} \wedge \cdot\right)}\right), \tau_{1}\right),\left(\left(X_{\left(\tau_{1}+\cdot\right) \wedge \tau_{2}}-X_{\tau_{1}}\right), \tau_{2}-\tau_{1}\right), \ldots,\left(\left(X_{\left(\tau_{k}+\cdot\right) \wedge \tau_{k+1}}-X_{\tau_{k}}\right), \tau_{k+1}-\tau_{k}\right)
$$

are independent variables under $Q_{0}$ and for $k \geqslant 1,\left(\left(X_{\left(\tau_{k}+\cdot\right) \wedge \tau_{k+1}}-X_{\tau_{k}}\right), \tau_{k+1}-\tau_{k}\right)$ are distributed like $\left(\left(X_{\tau_{1} \wedge}\right), \tau_{1}\right)$ under $P_{0}\left(\cdot \mid D_{\alpha}^{\ell_{0}}=\infty\right)$. 
Using again Lemma 3.2 .5 p. 265 in [10], we also have

Lemma 3. Under assumption $\left(\mathrm{H}^{\prime}\right)$,

$$
E_{Q_{0}}\left[X_{\tau_{1}} \cdot \ell \mid D_{\alpha}^{\ell}=\infty\right]=\frac{1}{P_{0}\left(D_{\alpha}^{\ell}=\infty\right)} .
$$

We have now all the tools to follow the proof of Theorem 1 except that $Q_{0}$ replaces $P_{0}$. We obtain the existence of $v_{0}$ satisfying (18).

\section{Acknowledgements}

I wish to thank my Ph.D. supervisor Francis Comets for his help and suggestions. I am also grateful to the anonymous referee for his remarks and comments.

\section{References}

[1] N. Berger, On the limiting velocity of high-dimensional random walk in random environment, arXiv: math.PR/0601656.

[2] F. Comets, O. Zeitouni, A law of large numbers for random walks in random mixing environments, Ann. Probab. 32 (1B) (2004) 880-914.

[3] F. Comets, O. Zeitouni, Gaussian fluctuations for random walks in random mixing environments, Israel J. Math. 148 (2005) $87-114$.

[4] S.A. Kalikow, Generalized random walk in a random environment, Ann. Probab. 9 (5) (1981) 753-768.

[5] H. Kesten, The limit points of a normalized random walk, Ann. Math. Statist. 41 (1970) 1173-1205.

[6] A.-S. Sznitman, An effective criterion for ballistic behavior of random walks in random environment, Probab. Theory Related Fields 122 (4) (2002) 509-544.

[7] A.-S. Sznitman, On new examples of ballistic random walks in random environment, Ann. Probab. 31 (1) (2003) $285-322$.

[8] A.-S. Sznitman, M. Zerner, A law of large numbers for random walks in random environment, Ann. Probab. 27 (4) (1999) $1851-1869$.

[9] H. Thorisson, Coupling, Stationarity, and Regeneration, Probability and its Applications (New York), Springer-Verlag, New York, 2000.

[10] O. Zeitouni, Random walks in random environment, in: Lectures on Probability Theory and Statistics, in: Lecture Notes in Math., vol. 1837, Springer, Berlin, 2004, pp. 189-312.

[11] M.P.W. Zerner, F. Merkl, A zero-one law for planar random walks in random environment, Ann. Probab. 29 (4) (2001) $1716-1732$. 